Supporting Information for

\title{
The Curious Case of Aqueous Warfarin: Structural Isomers or Distinct Excited States?
}

Sushil S. Sakpal ${ }^{\S \#}$, Deborin Ghosh ${ }^{\S \#}$, Meghna A. Manae ${ }^{\dagger}$, Anirban Hazra*†, and Sayan Bagchi*\$:

§Physical and Materials Chemistry Division, CSIR-National Chemical Laboratory, Pune 411008, India

\#Academy of Scientific and Innovative Research (AcSIR), Ghaziabad- 201002, India †Department of Chemistry, Indian Institute of Science Education and Research, Pune 411008, India.

\#Equal contribution

*ahazra@,iiserpune.ac.in, s.bagchi@ncl.res.in 


\section{Table of Contents}

1. Structure of warfarin anion $\quad$ S2

2. Absorption spectra of warfarin S3

3. Pyranocoumarin in acidic $\mathrm{pH}$ solution $\quad \mathrm{S} 3$

4. Absorption spectra of pyranocoumarin $\quad$ S3

5. Quantum chemically calculated transition energies $\quad$ S4

6. Identifying the highest occupied $n$ orbital for protonated warfarin S5

7. Identifying the highest occupied $n$ orbital for deprotonated warfarin S6

8. Calculated emission parameters of warfarin $\quad$ S6

9. Absorption and emission spectra of warfarin-HSA complex $\quad$ S7

\section{Structure of warfarin anion}

According to Figure 1 in the main manuscript, there are two possible deprotonated forms of warfarin (V and VI). However, during geometry optimization using DFT B3LYP/6$31+G(d, p)$, both structures converged to the same geometry. This convergence can be explained considering the conjugation between the hydroxyl oxygen and the ester carbonyl oxygen, which allows the negative charge to be equally distributed between two oxygen atoms.

\section{Optimized geometry of V and VI:}

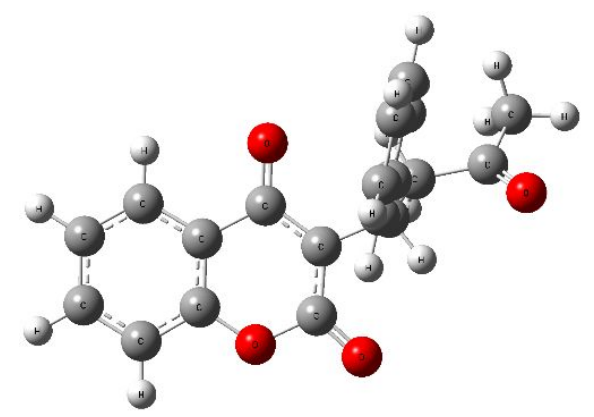




\section{Absorption spectra of warfarin}

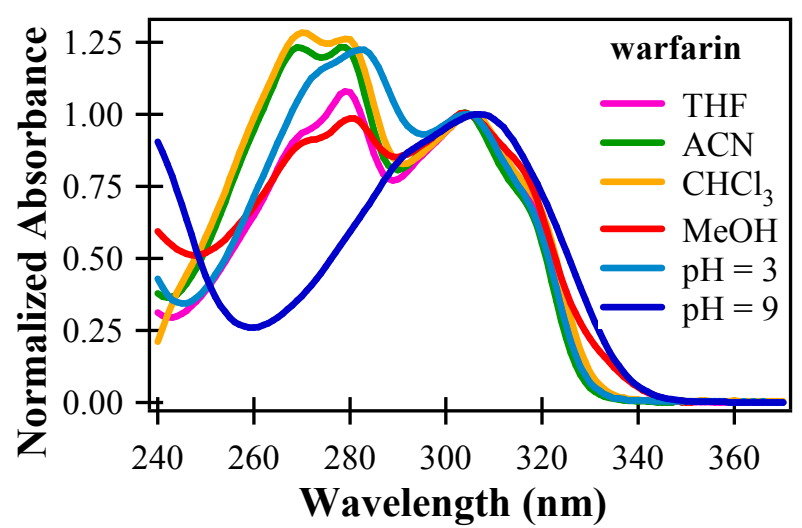

Figure S1. Normalized absorption spectra of warfarin in different non-aqueous solvents and aqueous solutions of acidic $(\mathrm{pH}=3)$ and basic $(\mathrm{pH}=9) \mathrm{pH}$. Similar spectra in nonaqueous solvents have been previously used as the basis of structural assignment of warfarin. (J. Phys. Chem. B 2007, 111, 10520-10528.)

\section{Pyranocoumarin in acidic $\mathbf{p H}$ solution}

One possible general reaction mechanism is shown below. In acidic $\mathrm{pH}$, the oxygen atom present in the cyclic ring of pyranocoumarin can capture a proton. The formation of oxonium ion can then take place through the involvement of the lone pair of electrons of the methoxy group which causes the breaking of the bond between the ring oxygen atom and the carbon atom attached to the methoxy group. Subsequent attack of water on the carbon atom of the oxonium ion can form open chain structure of warfarin.

\section{General reaction mechanism:}

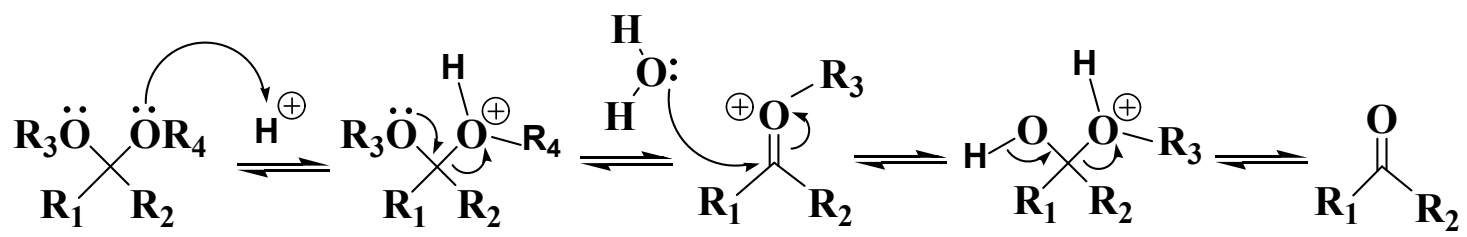

\section{Absorption spectra of pyranocoumarin}

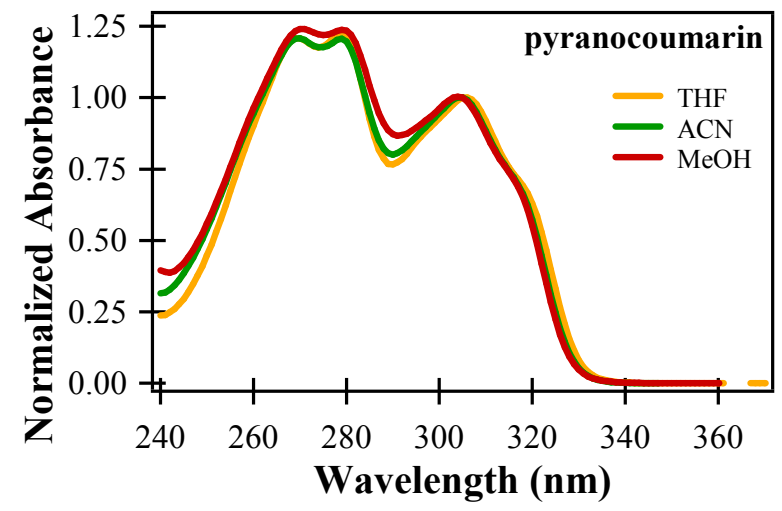

Figure S2. Normalized absorption spectra of pyranocoumarin in three non-aqueous solvents. 
5. Quantum chemically calculated transition energies

(a)

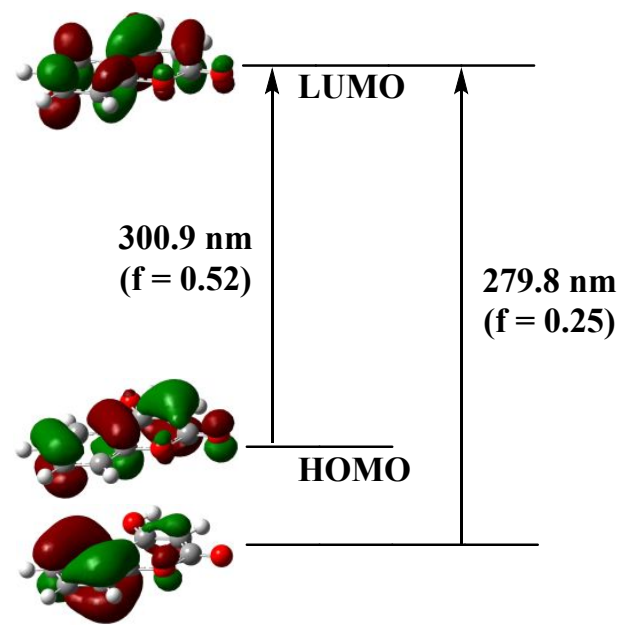

(c)

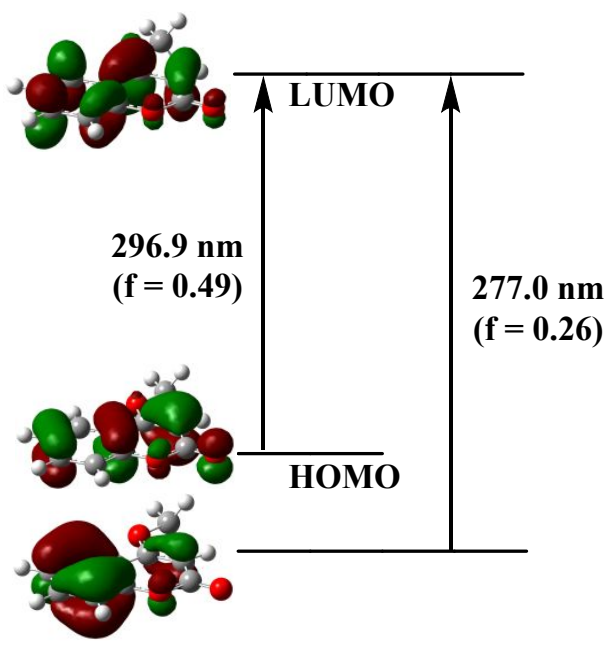

(e)

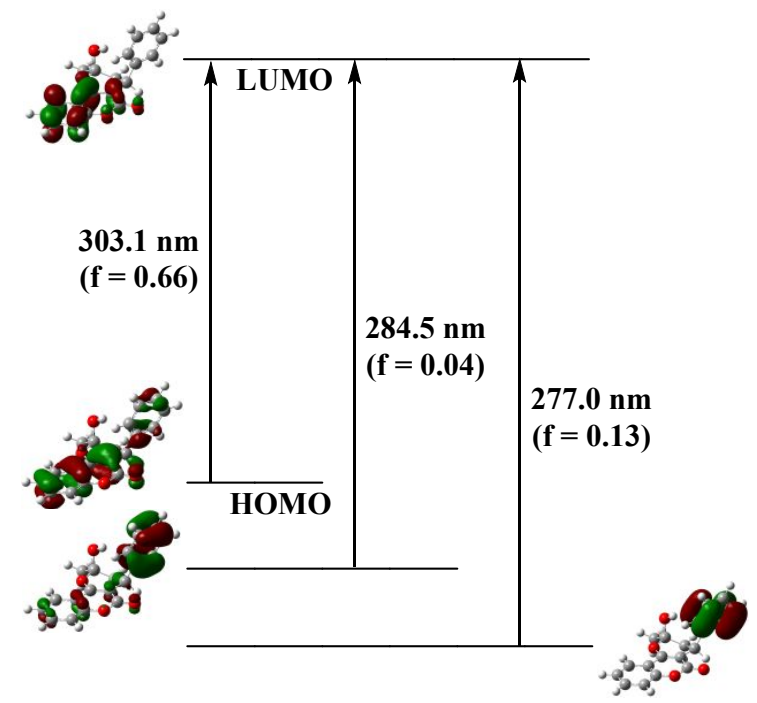

(b)

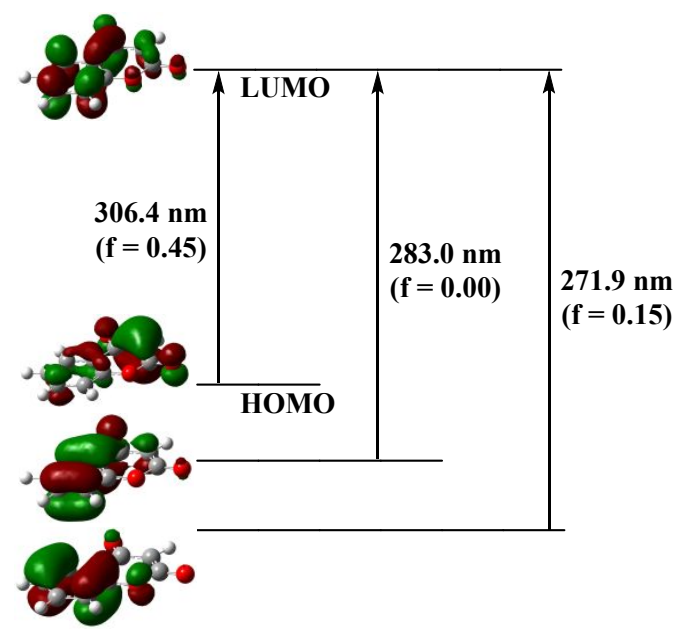

(d)

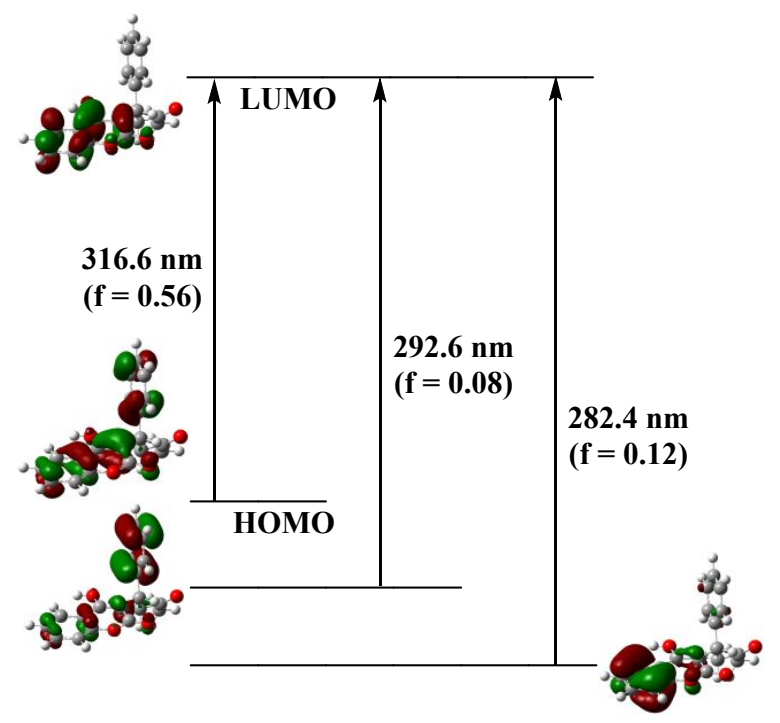

(f)

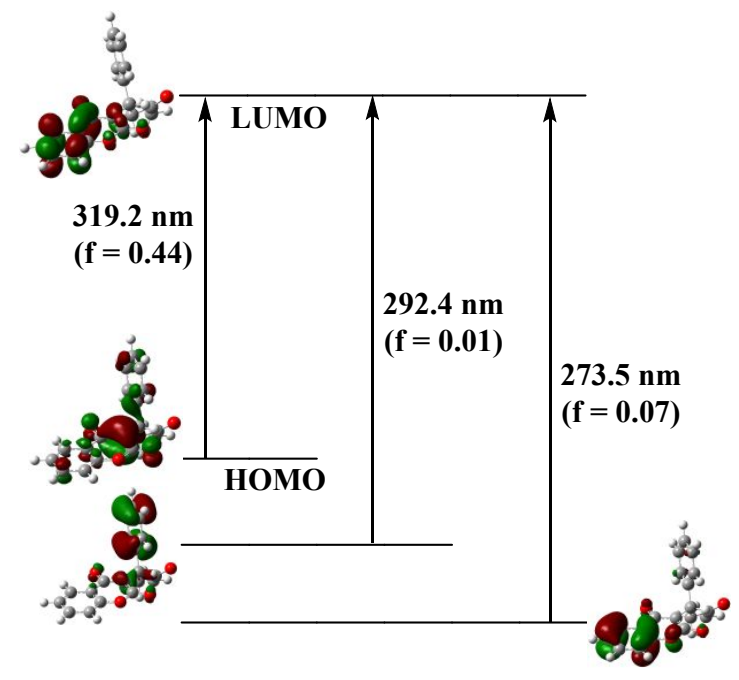

Figure S3. Energy level diagrams for (a) 4-hydroxycoumarin and (b) its anion; (c) 4methoxycoumarin, (d) open-chain form, (e) cyclic hemiketal form and (f) anion of warfarin in water (SMD solvation model). Molecular orbitals are calculated at the HF/6-31+G(d,p) level of theory 
while the excitation energies are calculated using the TDDFT B3LYP/6-31+G(d,p) method.

6. Identifying the highest occupied $\boldsymbol{n}$ orbital for protonated warfarin

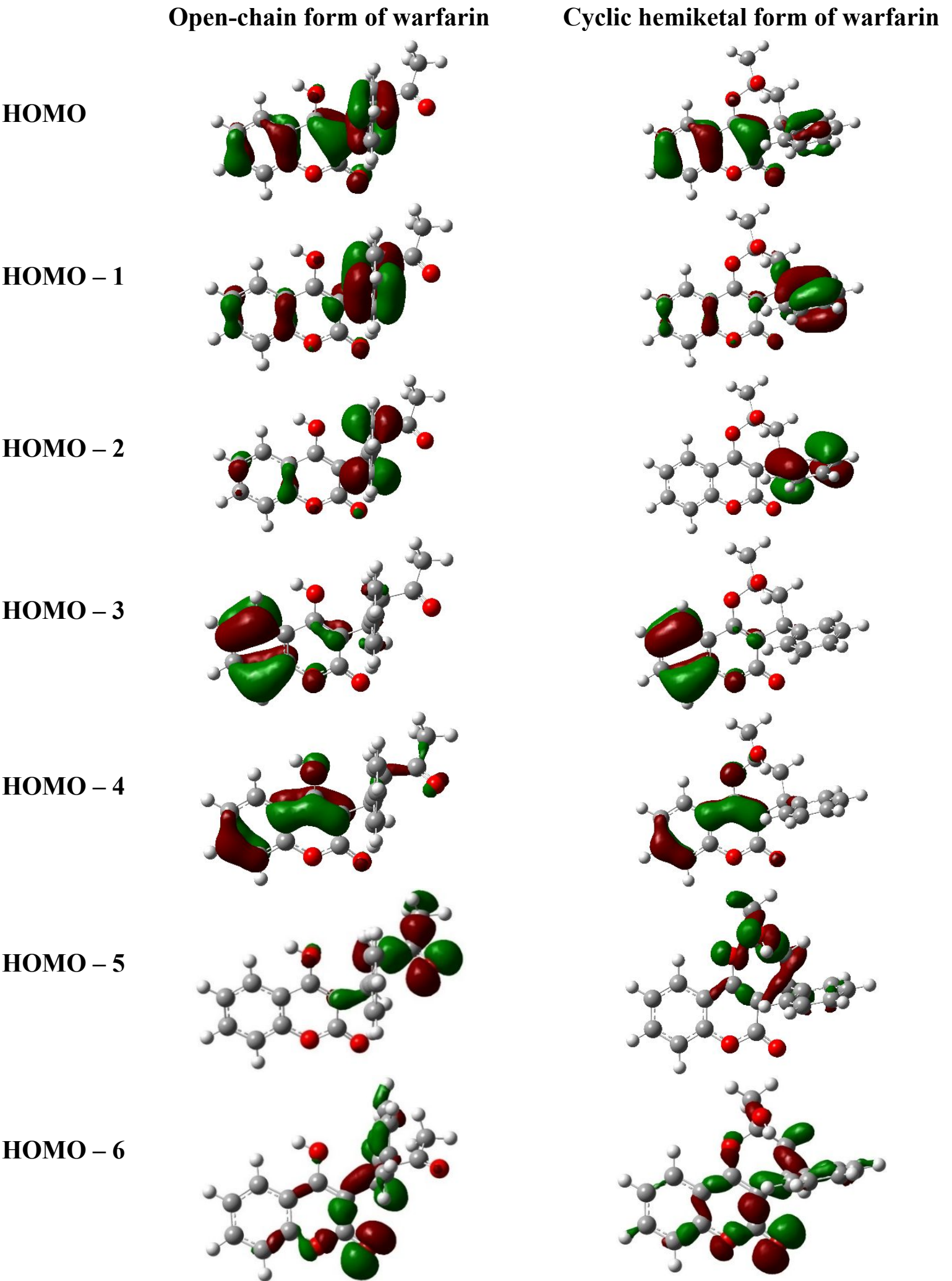

Figure S4. Some occupied molecular orbital picture (calculated at the HF/6-31+G(d,p) level of theory) of open-chain and hemiketal form of warfarin in water (SMD solvation model). Molecular orbital dominated by $n$ character appears HOMO -5 and HOMO -6 energy level 
respectively for open-chain and hemiketal form.

7. Identifying the highest occupied $\boldsymbol{n}$ orbital for deprotonated warfarin

HOMO

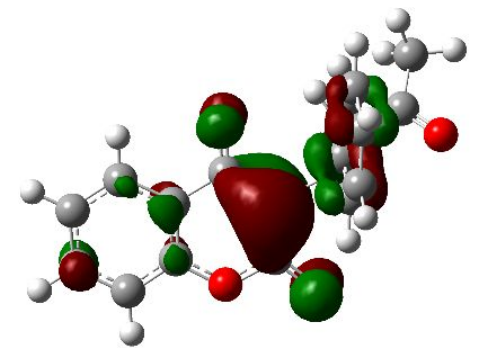

HOMO - 4

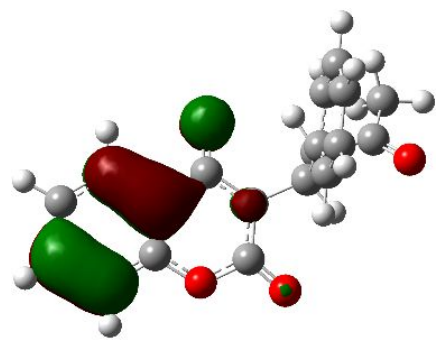

HOMO - 1

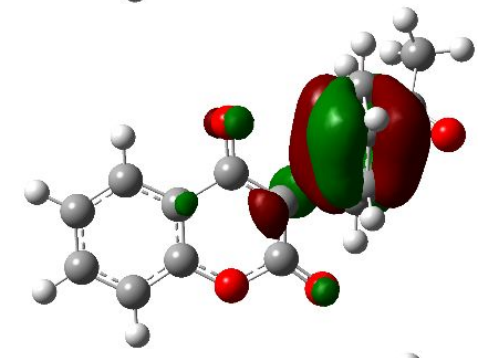

HOMO - 5

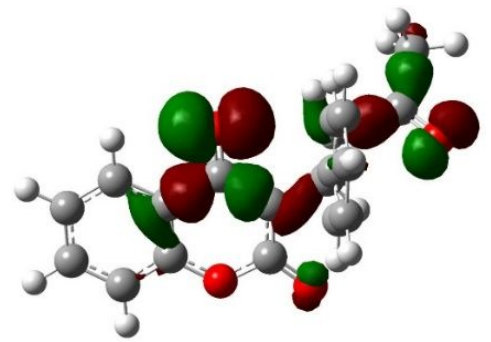

HOMO - 2

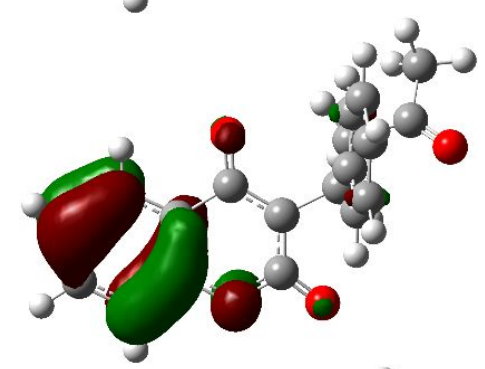

HOMO - 6
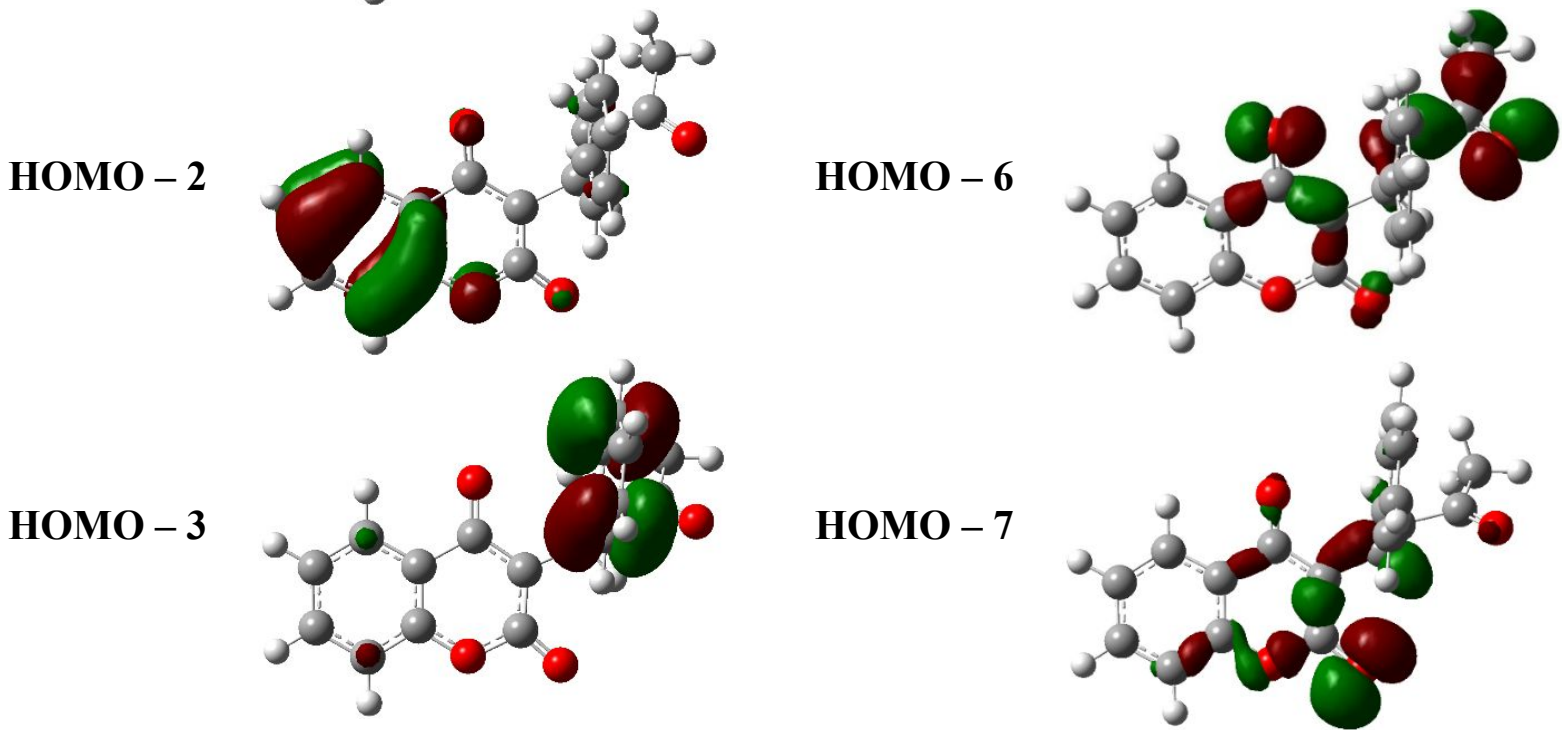

Figure S5. Some occupied molecular orbital picture (calculated at the HF/6-31+G(d,p) level of theory) of deprotonated form of warfarin in water (SMD solvation model). Molecular orbital dominated by $n$ character appears from HOMO -5 energy level.

\section{Calculated emission parameters of warfarin}

\begin{tabular}{|c|c|c|c|}
\hline Structure & Open Chain (I) & Hemiketal (III) & Anion \\
\hline Calculated $\lambda_{\mathrm{em}}(\mathrm{nm})$ & 341.1 & 349.0 & 374.0 \\
\hline Oscillator strength (f) & 0.72 & 0.69 & 0.42 \\
\hline Experimental $\lambda_{\text {em }}(\mathrm{nm})$ & \multicolumn{2}{|c|}{356} & 392 \\
\hline
\end{tabular}




\section{Absorption and emission spectra of warfarin-HSA complex}
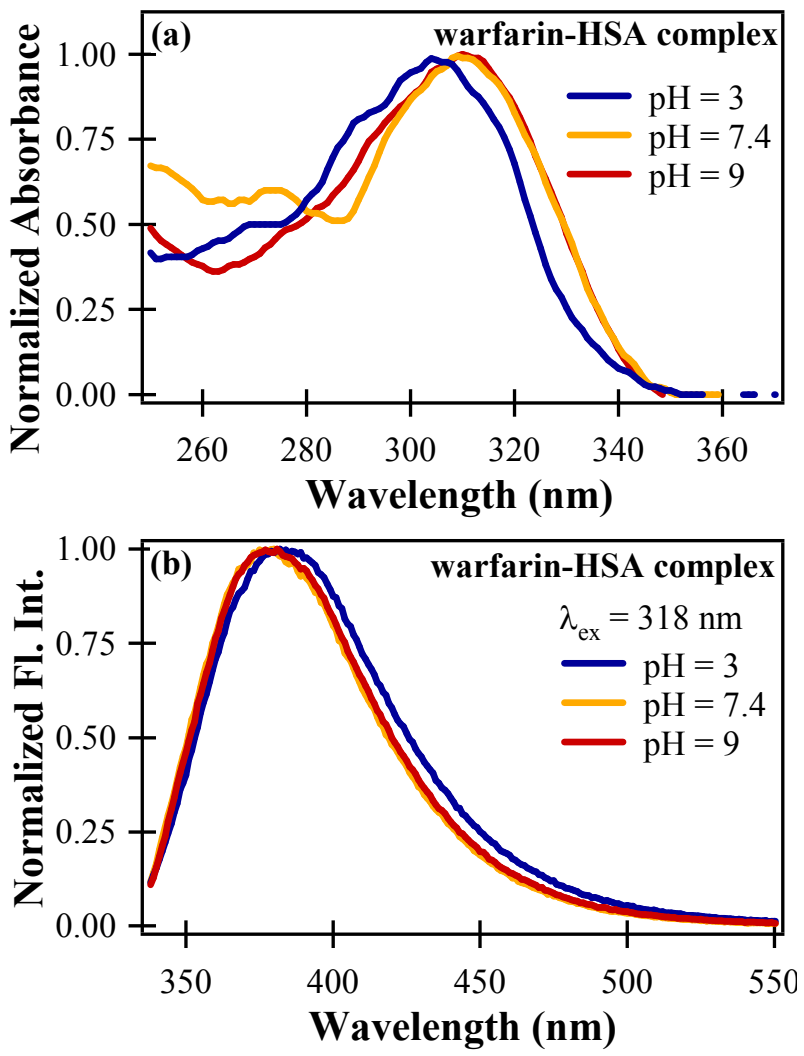

Figure S6. (a) Normalized absorption spectra of warfarin-HSA complex in aqueous solutions of different $\mathrm{pH}, \mathrm{HSA}$ solutions in corresponding $\mathrm{pH}$ were used as reference during UV-Vis absorption experiments. (b) Normalized emission spectra of warfarin-HSA complex in aqueous solutions of different $\mathrm{pH}\left(\lambda_{\mathrm{ex}}=318 \mathrm{~nm}\right)$. 